\title{
Rigid-flexible Coupling Analysis of Backhoe Hydraulic Excavator Working Device
}

\author{
Yongliang Yuan \\ School of Mechanical Engineering, Dalian University of Technology, Dalian 116024, China
}

\begin{abstract}
This paper develops a rigid-flexible coupling approach to predict the dynamic performance of the excavator, including the stress distribution and stress diagram of curves. In order to study the dynamic performance of the excavator during the mining process, the rigid-flexible coupling model of a excavator is established and carried out for dynamics simulation. The results show that the strength of excavator meets the expected requirement and there is a large optimization space. In order to reduce the mass of the excavator's boom, this paper optimizes the design of the boom. Based on the results of the optimization design, the rigid-flexible coupling simulation of the excavator was performed again, which verified that the strength of the boom is sufficient. Simulation results show that the virtual prototype software can effectively predict the dynamic performance of the excavator. This paper provides a solid foundation for further study of the lightweight design of the whole excavator component.
\end{abstract}

\section{Introduction}

Excavators are widely used as primary production equipment in surface mining operations, the dynamic response of the excavator reflects its performance. In order to improve the dynamic performance of the excavator, it is necessary to build the excavator model based on the virtual prototype technology just as to build a physical prototype. Li et al [1]. conducted an excavator model confirmed its feasibility with the virtual prototype software and obtained its kinematics principle. K.Awuah-Offei et al [2]. proposed the numerical method and obtained the lifting speed and thickness of cutting relational curves. Ma used Matlab/Simulink to establish the model and carry out the simulation [3]. He et al. [4] used the rigid rod to replace the steel wire rope to establish the virtual prototype model, and carried out the simulation. From the above literature, it can be seen that scholars' researches on excavators mainly focus on the study of movement laws and stress distribution, and there is less research on the light weighting of excavators. In order to improve the dynamic performance of the excavator, this paper optimizes the design of the excavator's boom. The rest parts of this paper are organized as follows: Section 2 discusses the simulation process of virtual prototype technology. Section 3 discusses the simulation results. The conclusion is presented in Section 4.

\section{Simulation process of virtual prototype technology}

Conventional design process uses a laboratory test to simulate the physical prototype to evaluate the performance of the excavator. Adams simulation of virtual prototype technology in product design has gradually become a major tool. Modern design processes in virtual simulation environments enable the testing of the excavator performance prior to final design and practical application. The virtual prototype technology not only reduces costs the product development cycle, but also predicts the performance of products and provides the basis for further optimizing product. Technology of virtual prototype simulation process is shown in Fig 1.

Step 1 Build the 3D model by CAD

In view of the fact that the ADAMS software establishes the excavator model is very difficult and has many limitations. The three-dimensional (3D) dynamic model is established by the third-party CAD software. In this study, the $3 \mathrm{D}$ dynamic model is saved as parasolid (.x_t) format and imported into the software of Automatic Dynamic Analysis of Mechanical Systems (ADAMS), and completed the rigid body model the establishment.

Step 2 Add the material property and constraints

The material property of the parts are added, according to the actual working condition. In accordance with the system actual working state to add restrictions and drive components.

Step 3 The establishment of a flexible body

In order to carry on the rigid-flexible coupling simulation, in this study, the flexible body is obtained by using the finite element software for modal analysis of the model. Using the ADAMS/Flex body module connection to import the file (.mnf) into ADAMS, finally we obtain the system rigid-flexible coupling model.

Step 4 Select the solver of ADAMS

\footnotetext{
* Corresponding author: yuan-yong-liang@163.com
} 


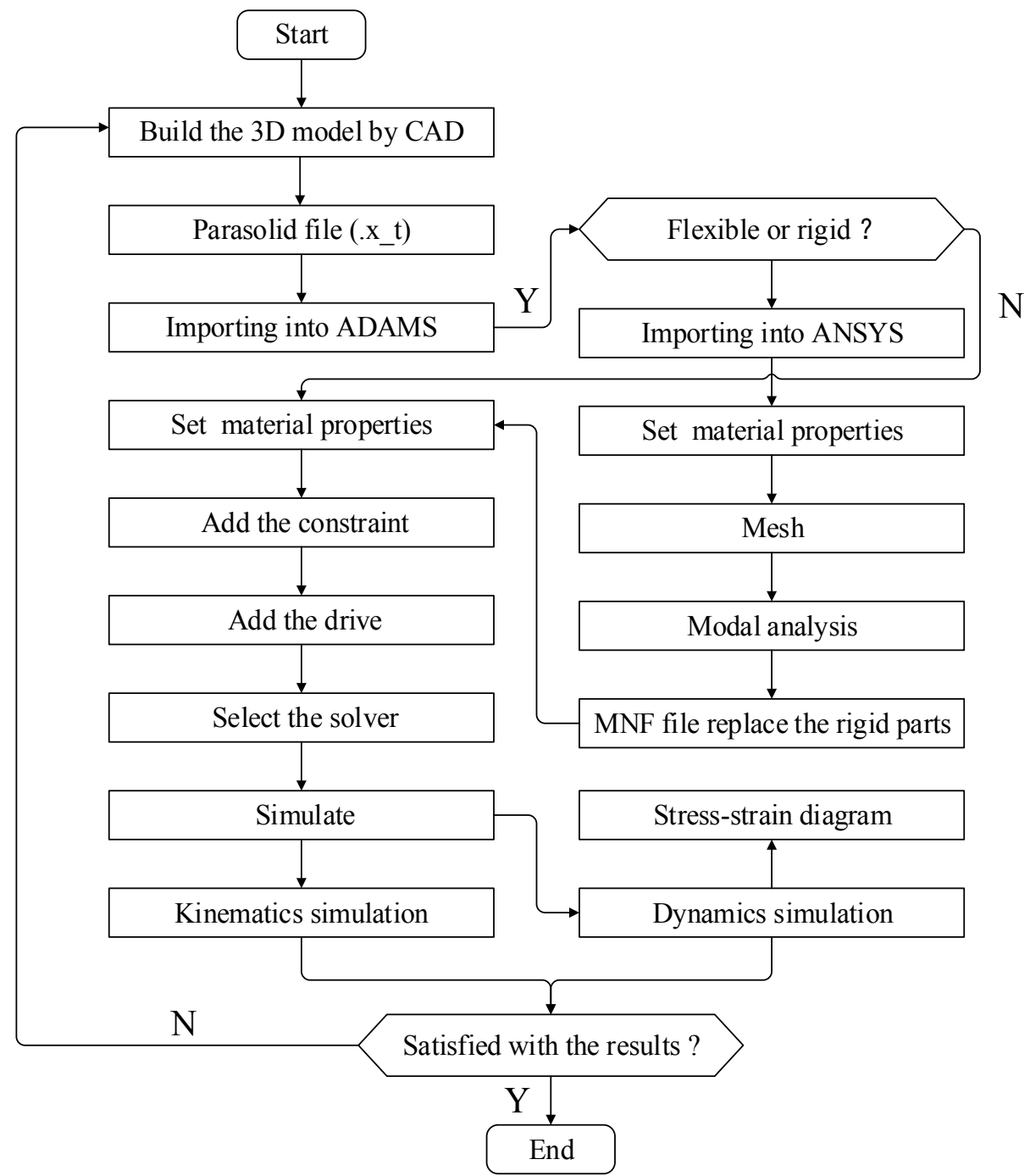

Fig. 1. The Simulation Process of Virtual Prototype.

In view of the fact that the ADAMS software has many solvers, in order to obtain the accurate results, it becomes very important to select the appropriate solver and the integration method. To solve the differential equations of the motion, we also choose the appropriate step length of the coupled simulation.

Step 5 Simulation Results \& Analysis

After the simulation, using the ADAMS postprocessing module to carry on the simulation result, we can obtain the motion law of system and the dynamics curve. Finally, we can grasp its dynamics performance.

\section{Rigid-flexible coupling simulation analysis}

\subsection{Pre-optimization simulation}

The mechanism used in this case has the same characteristics as previously, except that the boom and dipper handle. The boom and dipper handle are saved as the need neutral file (.mnf) and import into the Adams to obtain the rigid-flexible coupling model of excavator. In order to ensure the authenticity of the excavator model, the model of the material, constraints, and parameters are set according to the actual situation[5-8]. This paper chooses the integration tolerance is 0.001 , the simulation time is $16 \mathrm{~s}$ and the step size is $0.02 \mathrm{~s}$.

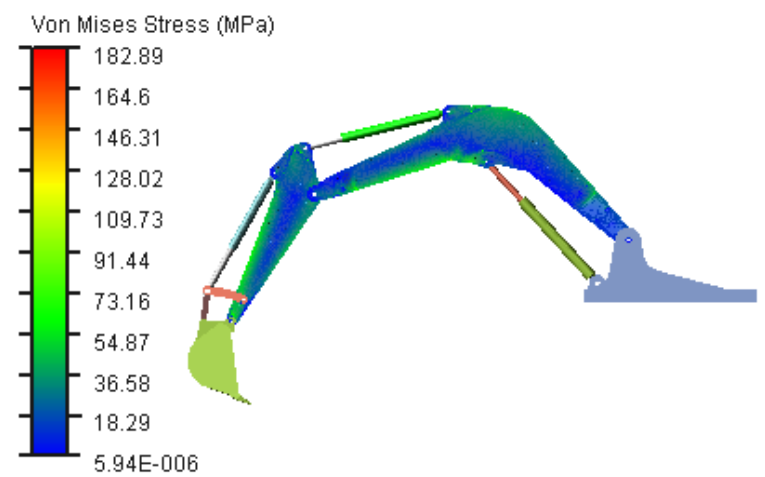

Fig. 2. The Stress Distribution of Excavator.

As can be seen from Figure 2, the maximum stress of the excavator is $182.89 \mathrm{Mpa}$, and the maximum stress stress occurs at the hinge hole of the boom of the excavator. The main reason for this is the uneven force exerted by the excavator during excavation. From the value of the maximum stress, it can be known that the strength of the excavator can meet the design requirements and the large arm has a large optimization space. 


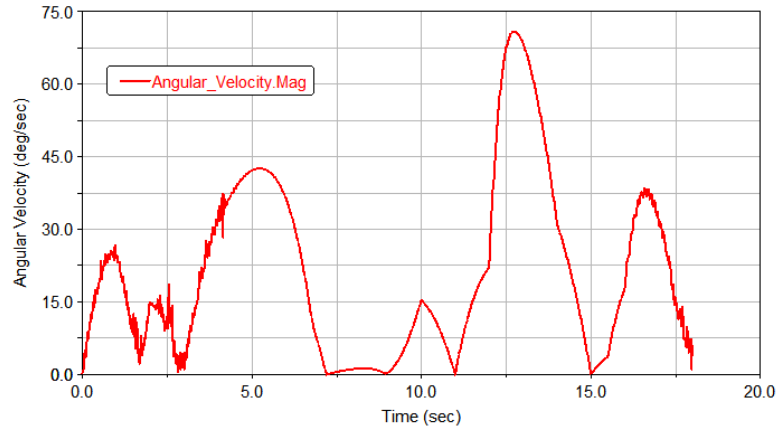

Fig. 3. The Angular Velocity of Arm and Lifting Cylinder Hinge.

As shown in Figure 3, the angular velocity of arm and lifting cylinder hinge is $72.8 \mathrm{deg} / \mathrm{s}$. It can be seen from the figure that the excavator does not work smoothly during the work, but there is some vibration. As the excavation material increases, the vibration of the excavator decreases and gradually stabilizes.

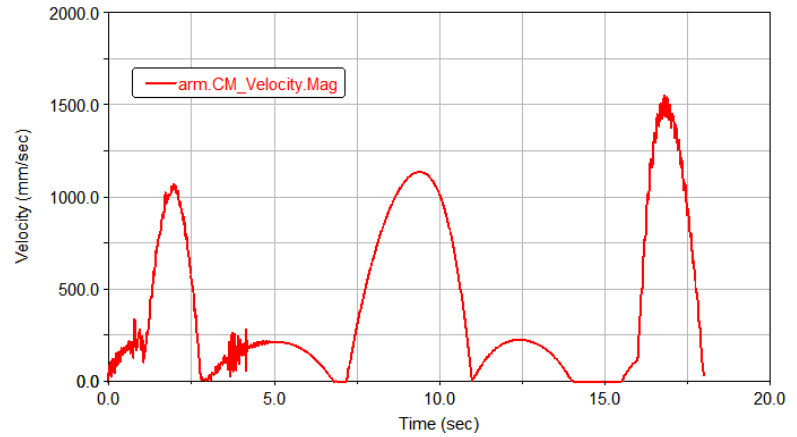

Fig. 4. The Speed of Arm

Figure 4 illustrates that there is also some vibration in the whole work process of the arm, which is similar to the reason of Figure 3. The maximum value is $1547.2 \mathrm{~mm} / \mathrm{s}$. After the excavator dumped the material, the excavator began to vibrate again. In order to reduce the vibration of the excavator, reduce the speed of the hydraulic cylinder as much as possible while satisfying the excavation requirements.

\subsection{Optimization simulation}

The analysis of section 2 shows that the strength of the arm is sufficient. In order to reduce the weight of the excavator, this paper optimizes the lightweight design of the boom. Combined with the optimized results, the dynamics simulation was performed again. By comparing with the previous results, the influence of the dynamic performance of the excavator was obtained.

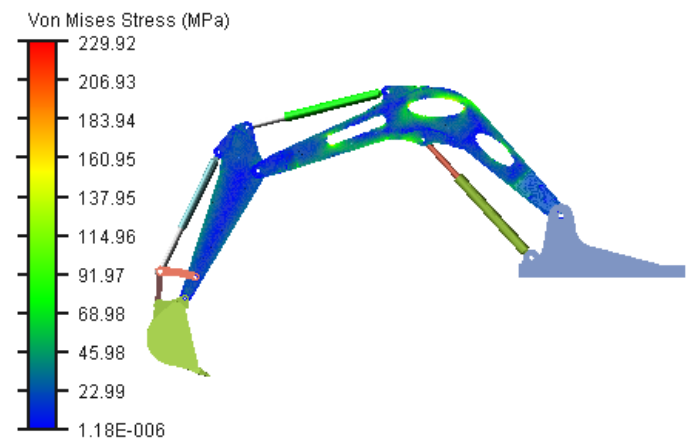

Fig. 5. Optimized Stress Distribution.
Figure 5 shows that the stress of the excavator after the optimization of the boom is greater than the stress before the optimization, mainly because the weight of the boom becomes smaller. The maximum stress after optimization is $229.92 \mathrm{MPa}$, which is $47.03 \mathrm{MPa}$ larger than before optimization. Although the optimized stress is larger, it still meets the strength requirements. So we can continue to optimize the design based on this result.

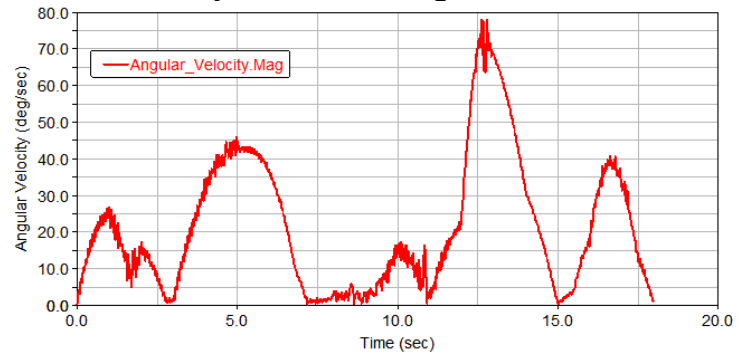

Fig. 6. The Angular Velocity of Arm and Lifting Cylinder Hinge of Optimized Result.

By comparing Figure 3 and Figure 6, it can be seen that the optimized maximum value is $72.8 \mathrm{deg} / \mathrm{s}$. Figure 6 shows that the optimized vibration is more pronounced than before the optimization, which is mainly due to the lower weight of the big arm and the shift of the centroid. In order to reduce the vibration of the excavator, reduce the speed of the hydraulic cylinder as much as possible while satisfying the excavation requirements.

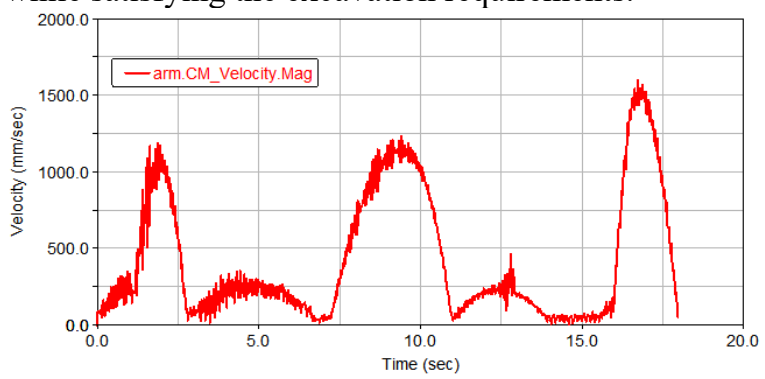

Fig. 7. The Speed of Arm of Optimized Result.

Figure 7 illustrates that there is also some vibration in the whole work process of the arm, which is similar to the reason of Figure 6 . The maximum value is $1631.9 \mathrm{~mm} / \mathrm{s}$. After the excavator dumped the material, the excavator began to vibrate again. Through the optimization analysis of the boom of the excavator, it can be seen that as the mass of the boom decreases, the vibration of the excavator increases significantly. In order to reduce the vibration of the excavator, it should be optimized from the whole to avoid resonance between the components and the excavator.

\section{Conclusion}

This paper uses virtual prototyping to verify the necessity of rigid-flexible coupled simulation of excavator. First, we establish the model which is the rigid body dynamics simulation of excavator by UG. The modal analysis is carried out on the boom and the dipper handle and the modal neutral file is got at the same time. Second, the stress distributions of the boom and dipper handle are obtained, including the pre-optimization simulation results and the optimized simulation. Third, by comparing the results before and after optimization, 
the results show that the virtual prototype software can effectively predict the dynamic performance of the excavator. In later studies, in order to make the performance of excavator to be the best, it' $s$ necessary that we should consider the boom and dipper handle are flexible body at the same time.

\section{References}

1. Y. Li, S. Frimpong. Int. J. Adv. Manuf. Tech. 37, 5 (2008).

2. K. Awuah-Offei, S. Frimpong. Mech. Mach. Theory. 42, 8, (2007)

3. M.H. Ma. Jilin University. (2009)

4. B. He, G. Zhou, S. Hou, et al. Int. J. Adv. Manuf. Tech. 89, 9, (2017)

5. Y.L. Yuan, L. Du, W. Sun, et al. International Conference on Mechanical Design, (2017)

6. X.L. Luo, A.T. Lewandowski, D. Berlin, et al. The First Int Colloq Microfluid, Shenyang, (2007)

7. W. Lin, G. Zhu, Y. Tang, et al. P. I. Mech. Eng. C-J. Mec. 229, 2, (2015)

8. S. Šalinić, G. Bošković, M. Nikolić. Automat. Constr. 44, (2014) 